PROCEEDINGS OF THE

AMERICAN MATHEMATICAL SOCIETY

Volume 139, Number 10, October 2011, Pages 3491-3496

S 0002-9939(2011)11170-5

Article electronically published on May 24, 2011

\title{
LIOUVILLE THEOREMS FOR THE ANCIENT SOLUTION OF HEAT FLOWS
}

\author{
MENG WANG \\ (Communicated by Michael T. Lacey)
}

\begin{abstract}
Let $M$ be a complete Riemannian manifold with Ricci curvature bounded from below: $\operatorname{Ric}(M) \geq-\kappa$. Let $N$ be a simply connected complete Riemannian manifold with nonpositive sectional curvature. Using a gradient estimate, we prove Liouville's theorem for the ancient solution of heat flows.
\end{abstract}

\section{INTRODUCTION}

In 1964, Eells and Sampson introduced the heat flow method to study the problem of existence of harmonic maps between compact Riemannian manifolds. Let $(M, g)$ be a compact Riemannian manifold and let $N$ be a closed submanifold of the Euclidean space $R^{k}$. They proved that, if the sectional curvature $K_{N}$ of $N$ is nonpositive, then any map $\phi \in C^{1}(M, N)$ can be deformed to a harmonic map. The heat equation of harmonic maps is the following parabolic system:

$$
\frac{\partial u}{\partial t}=\tau(u)=\Delta u+A(u)(\nabla u, \nabla u),
$$

where $\tau(u)$ is called the tension field of the mapping $u$, and $A(y): T_{y}(N) \times T_{y}(N) \rightarrow$ $\left(T_{y}(N)\right)^{\perp}$ is the second fundamental form of $N$ in $R^{k}$.

In [5, Li and Tam considered this equation in the case that $M$ is noncompact with Ricci curvature bounded from below and $N$ is a complete Riemannian manifold with nonpositive sectional curvature [5, Theorem 4.1].

If $N=R$, then $u$ is a function, and the equation (1.1) becomes the following heat equation:

$$
\frac{\partial u}{\partial t}=\Delta u
$$

As to this equation, Li-Yau [3] obtained the following parabolic gradient estimate.

Theorem A. Let $M$ be a complete manifold of dimension $m \geq 2$ with Ricci $(M) \geq$ $-\kappa$ for some $\kappa \geq 0$. Suppose that $u$ is any positive solution to the heat equation in $B\left(x_{0}, R\right) \times\left[t_{0}-T, t_{0}\right]$. Then for $a>1$,

$$
\frac{|\nabla u|^{2}}{u^{2}}-a \frac{u_{t}}{u} \leq \frac{c_{n}}{R^{2}}+\frac{c_{n}}{T}+c_{n} \kappa
$$

Received by the editors August 18, 2010.

2010 Mathematics Subject Classification. Primary 35K05, 58J35.

Key words and phrases. Liouville theorem, ancient solution, quasi-harmonic map, heat flow.

The author's research was partially supported by NSFC 10701064, 10931001.

(C) 2011 American Mathematical Society Reverts to public domain 28 years from publication 
in $B\left(x_{0}, R / 2\right) \times\left[t_{0}-T, t_{0}\right]$. Here $c_{n}$ depends only on the dimension $n$ and $a$.

For the time-dependent solution of the heat equation, it is well known that the elliptic Cheng-Yau type gradient estimate [1] cannot be true in general. The typical example is the function $u(x, t)=e^{-|x|^{2} / 4 t} /(4 \pi t)^{n / 2}$. However, in the case of compact manifolds, Hamilton's estimate shows that one can get a certain elliptic gradient estimate. In [6], the author obtained

Theorem B. Let $M$ be a compact manifold without boundary and with Ricci $(M) \geq$ $-\kappa$ for some $\kappa \geq 0$. Let $u$ be a smooth positive solution of the heat equation with $u \leq M$ for all $(x, t) \in M \times(0, \infty)$. Then

$$
\frac{|\nabla u|^{2}}{u^{2}} \leq\left(\frac{1}{t}+2 k\right) \ln \frac{M}{u} .
$$

It would be highly desirable to have a noncompact version of Hamilton's estimate. In 4, they discovered that the elliptic Cheng-Yau estimate actually holds for the heat equation, after inserting a necessary logarithmic correction term. Their main result is the following:

Theorem C. Let $M$ be a Riemannian manifold of dimension $n \geq 2$ with Ricci $(M)$ $\geq-\kappa$ for some $\kappa \geq 0$. Suppose that $u$ is any positive solution to the heat equation in $Q_{R, T} \equiv B\left(x_{0}, R\right) \times\left[t_{0}-T, t_{0}\right] \subset M \times(-\infty, \infty)$. Suppose also that $u \leq M$ in $Q_{R, T}$. Then there exists a dimensional constant $c$ such that

$$
\frac{|\nabla u(x, t)|}{u(x, t)} \leq c\left(\frac{1}{R}+\frac{1}{\sqrt{T}}+\sqrt{\kappa}\right)\left(1+\ln \frac{M}{u(x, t)}\right)
$$

in $Q_{R / 2, T / 2}$.

Moreover, if $M$ has nonnegative Ricci curvature and $u$ is any positive solution of the heat equation on $M \times(0, \infty)$, then there exist dimensional constants $c_{1}, c_{2}$ such that

$$
\frac{|\nabla u(x, t)|}{u(x, t)} \leq c_{1} \frac{1}{t^{1 / 2}}\left(c_{2}+\ln \frac{u(x, 2 t)}{u(x, t)}\right)
$$

for all $x \in M$ and $t>0$.

Remark 1.1. Without seeing the paper 4, we obtained the Liouville theorem for the positive quasi-harmonic function by a different method in one of our papers which is to appear. It is just the time-independent version of (1.5) for $M=R^{m}$.

The main purpose of this paper is to consider the elliptic gradient estimate for the ancient solution of the equation (1.1) when the target manifold $N$ is a general complete manifold. We can obtain that

Theorem 1.2. Let $M$ be a complete Riemannian manifold with Ricci curvature bounded from below: $\operatorname{Ric}(M) \geq-\kappa$. Let $N$ be a simply connected complete Riemannian manifold with nonpositive sectional curvature. Let $u$ be a solution of the equation (1.1). Assume that $y_{0} \notin u\left(B\left(x_{0}, R\right) \times\left[t_{0}-T, t_{0}\right]\right)$. Let $\rho(y)$ be the distance between $y$ and $y_{0}$ in $N$. Then, if $b>2 \sup \left\{\rho(u(x, t)) \mid(x, t) \in B\left(x_{0}, R\right) \times\left[t_{0}-T, t_{0}\right]\right\}$, we have

$$
\sup _{Q_{R / 2, T / 2}} \frac{|\nabla u|(x, t)}{b^{2}-\rho^{2}(u(x, t))} \leq \frac{C}{b}\left(\frac{1}{R}+\frac{1}{\sqrt{T}}+\sqrt{\kappa}\right),
$$

where $C>0$ depends only on $m$ and $N$. 
As a corollary of the above theorem, we can get the following Liouville theorem for the ancient solution of the equation (1.1) with certain growth conditions.

Fixing $p \in M$, let $d(x)$ be the distance between $x$ and $p$.

Theorem 1.3. Let $M$ be a complete, noncompact manifold with nonnegative curvature. Let $N$ be a simply connected Riemannian manifold with nonpositive sectional curvature. Let $u$ be an ancient solution to the equation (1.1) such that $\rho(u(x, t))=o(d(x)+\sqrt{|t|})$ near infinity. Then $u$ is a constant.

Remark 1.4. (1) If we consider a bounded quasi-harmonic map $v$ from $R^{m}$ to $N$, that is, $v$ is a bounded map satisfying

$$
\tau(v)=\frac{1}{2} x \cdot \nabla v
$$

then $u(x, t)=v\left(\frac{x}{\sqrt{-t}}\right)$ satisfies the assumption of Theorem 1.3. Thus the result of section 3 in 2] can be deduced by Theorem 1.3 .

(2) If $N=R$, Theorem 1.3 is [4, Theorem 1.2].

(3) Let $u(x, t)=x$ for $(x, t) \in R \times(-\infty, 0]$. Clearly it is an ancient solution for the heat equation and it is not constant. So our growth condition in the spatial direction is sharp in this sense.

\section{Proof of theorems}

The quantity we used to do a gradient estimate is $\frac{|\nabla u|^{2}}{b^{2}-\rho^{2}(u(x, t))}$, which is different from the quantity used in 4 .

Proof of Theorem 1.2, Let

$$
\phi(x, t)=\frac{|\nabla u|^{2}}{\left(b^{2}-\rho^{2}(u(x, t))\right)^{2}} .
$$

Then

$$
\nabla \phi(x)=\frac{\nabla\left(|\nabla u|^{2}\right)}{\left(b^{2}-\rho^{2}\right)^{2}}+2 \frac{|\nabla u|^{2} \nabla \rho^{2}}{\left(b^{2}-\rho^{2}\right)^{3}}
$$

and

$$
\Delta \phi(x)=\frac{\Delta\left(|\nabla u|^{2}\right)}{\left(b^{2}-\rho^{2}\right)^{2}}+4 \frac{\nabla\left(|\nabla u|^{2}\right) \cdot \nabla \rho^{2}}{\left(b^{2}-\rho^{2}\right)^{3}}+2 \frac{|\nabla u|^{2} \Delta \rho^{2}}{\left(b^{2}-\rho^{2}\right)^{3}}+6 \frac{\left|\nabla \rho^{2}\right|^{2}|\nabla u|^{2}}{\left(b^{2}-\rho^{2}\right)^{4}} .
$$

Note the following Bochner formula:

$$
\frac{1}{2} \Delta\left(|\nabla u|^{2}\right)=|\nabla d u|^{2}+\langle\nabla u, \nabla \tau(u)\rangle+\left\langle\operatorname{Ric}^{M} \nabla u, \nabla u\right\rangle-\left\langle R^{N}\left(u_{i}, u_{j}\right) u_{j}, u_{i}\right\rangle,
$$

where $u_{i}=d u\left(e_{i}\right)$ and $e_{1}, e_{2}, \ldots, e_{n}$ is an orthonormal frame of $M$. Using the assumption of Theorem 1.2, we have

$$
\Delta|\nabla u|^{2} \geq 2|\nabla d u|^{2}+\partial_{t}|\nabla u|^{2}-\kappa|\nabla u|^{2} .
$$

A direct calculation leads to

$$
\Delta \rho^{2}(u(x, t))=H\left(\rho^{2}\right)(\nabla u, \nabla u)+\partial_{t}\left(\rho^{2}(u(x, t))\right),
$$

where $H\left(\rho^{2}\right)$ is the Hessian of $\rho^{2}$. Since the sectional curvature of $N, K_{N} \leq 0$, the Hessian comparison theorem implies that

$$
\Delta\left(\rho^{2}(u(x, t))\right) \geq 2|\nabla u|^{2}+\partial_{t}\left(\rho^{2}(u(x, t))\right) .
$$


Then we have

$$
\begin{aligned}
\Delta \phi(x) \geq & \frac{2|\nabla d u|^{2}+\partial_{t}|\nabla u|^{2}-\kappa|\nabla u|^{2}}{\left(b^{2}-\rho^{2}\right)^{2}}+4 \frac{\nabla \rho^{2} \cdot \nabla\left(|\nabla u|^{2}\right)}{\left(b^{2}-\rho^{2}\right)^{3}} \\
& +\frac{4|\nabla u|^{4}+2 \partial_{t}\left(\rho^{2}(u(x, t))\right)|\nabla u|^{2}}{\left(b^{2}-\rho^{2}\right)^{3}}+\frac{6\left|\nabla \rho^{2}\right|^{2}|\nabla u|^{2}}{\left(b^{2}-\rho^{2}\right)^{4}}
\end{aligned}
$$

and

$$
\partial_{t} \phi=\frac{\partial_{t}|\nabla u|^{2}}{\left(b^{2}-\rho^{2}\right)^{2}}+2 \frac{|\nabla u|^{2} \partial_{t} \rho^{2}}{\left(b^{2}-\rho^{2}\right)^{3}} .
$$

Note that

$$
\begin{aligned}
\frac{2|\nabla d u|^{2}}{\left(b^{2}-\rho^{2}\right)^{2}}+\frac{2\left|\nabla \rho^{2}\right|^{2}|\nabla u|^{2}}{\left(b^{2}-\rho^{2}\right)^{4}} & \geq 4 \frac{|\nabla d u||\nabla u|\left|\nabla \rho^{2}\right|}{\left(b^{2}-\rho^{2}\right)^{3}} \\
& \geq 2 \frac{\left.|\nabla| \nabla u\right|^{2}|| \nabla \rho^{2} \mid}{\left(b^{2}-\rho^{2}\right)^{3}}
\end{aligned}
$$

and

$$
\frac{\nabla \rho^{2} \cdot \nabla \phi}{b^{2}-\rho^{2}}=\frac{\nabla \rho^{2} \cdot \nabla\left(|\nabla u|^{2}\right)}{\left(b^{2}-\rho^{2}\right)^{3}}+2 \frac{\left|\nabla \rho^{2}\right|^{2}|\nabla u|^{4}}{\left(b^{2}-\rho^{2}\right)^{4}} .
$$

By (2.3), (2.4), (2.5) and (2.6), we have

$$
\Delta \phi-\partial_{t} \phi \geq \frac{4|\nabla u|^{4}}{\left(b^{2}-\rho^{2}\right)^{3}}-\kappa \frac{|\nabla u|^{2}}{\left(b^{2}-\rho^{2}\right)^{2}}+2 \frac{\nabla \phi \cdot \nabla \rho^{2}}{b^{2}-\rho^{2}} .
$$

From here, we will use the well-known cutoff function of Li-Yau [3] and the argument in 4 .

Let $\psi=\psi(x, t)$ be a smooth cutoff function supported in $Q_{R, T}$, satisfying the following properties:

(1) $\psi=\psi\left(d\left(x, x_{0}\right), t\right) \equiv \psi(r, t) ; \psi(x, t)=1$ in $Q_{R / 2, T / 4}, 0 \leq \psi \leq 1$.

(2) $\psi$ is decreasing as a radial function in the spatial variables.

(3) $\left|\partial_{r} \psi\right| / \psi^{a} \leq C_{a} / R,\left|\partial_{r}^{2} \psi\right| / \psi^{a} \leq C_{a} / R^{2}$ when $0<a<1$.

(4) $\left|\partial_{r} \psi\right| / \psi^{1 / 2} \leq C / T$.

Let $L=-2 \frac{\nabla \rho^{2}}{b^{2}-\rho^{2}}$. We can calculate

$$
\begin{aligned}
& \Delta(\psi \phi)+L \cdot \nabla(\psi \phi)-2 \frac{\nabla \psi}{\psi} \cdot \nabla(\psi \phi)-(\psi \phi)_{t} \\
& \quad=\psi\left(\Delta \phi-\phi_{t}\right)+\phi\left(\Delta \psi-\psi_{t}\right)+\psi L \cdot \nabla \phi+\phi L \cdot \nabla \psi-2 \frac{|\nabla \psi|^{2}}{\psi} \phi \\
& \quad \geq \psi\left(4 \frac{|\nabla u|^{4}}{\left(b^{2}-\rho^{2}\right)^{3}}-\kappa \frac{|\nabla u|^{2}}{\left(b^{2}-\rho^{2}\right)^{2}}\right)+\phi\left(\Delta \psi-\psi_{t}\right)-2 \frac{\phi \nabla \rho^{2} \cdot \nabla \psi}{b^{2}-\rho^{2}}-2 \frac{|\nabla \psi|^{2}}{\psi} \phi .
\end{aligned}
$$

Suppose that the maximum of $\psi \phi$ is reached at $\left(x_{1}, t_{1}\right)$. By [3], $x_{1}$ is not in the cut locus of $M$. Then at this point, one has

$$
\Delta(\psi \phi) \leq 0,(\psi \phi)_{t} \geq 0, \text { and } \nabla(\psi \phi)=0 .
$$


So, we have at $\left(x_{1}, t_{1}\right)$,

$$
\begin{aligned}
& 4 \psi \frac{|\nabla u|^{4}}{\left(b^{2}-\rho^{2}\right)^{3}}-\kappa \psi \frac{|\nabla u|^{2}}{\left(b^{2}-\rho^{2}\right)^{2}} \\
& \quad \leq 2 \phi \frac{\nabla \rho^{2} \cdot \nabla \psi}{b^{2}-\rho^{2}}+2 \phi \frac{|\nabla \psi|^{2}}{\psi}+\phi\left(\psi_{t}-\Delta \psi\right) \\
& \quad=I+I I+I I I .
\end{aligned}
$$

Using the fact that $\left|\nabla \rho^{2}\right| \leq b|\nabla u|$, we can get

$$
\begin{aligned}
I & \leq 2|\phi| \frac{\left|\nabla \rho^{2}\right|}{b^{2}-\rho^{2}}|\nabla \psi| \\
& \leq 2 b|\phi| \frac{|\nabla u|}{b^{2}-\rho^{2}}|\nabla \psi| \\
& \leq 2 b|\phi|^{3 / 2}|\nabla \psi| \leq b^{2}\left|\psi \phi^{2}\right|+C \frac{|\nabla \psi|^{4}}{b^{2} \psi^{3}} .
\end{aligned}
$$

By the properties of $\psi$, one has

$$
I I \leq \frac{b^{2} \psi \phi^{2}}{8}+\frac{C}{b^{2} R^{4}} .
$$

As for $I I I=\phi\left(\psi_{t}-\Delta \psi\right)$, by the properties of $\psi$ and the assumption on the Ricci curvature, we have that

$$
\begin{aligned}
-\phi \Delta \psi & \leq-\left(\partial_{r}^{2} \psi+(m-1) \frac{\partial_{r} \psi}{r}+\sqrt{\kappa}\left|\partial_{r} \psi\right|\right) \phi \\
& \leq \frac{1}{8} b^{2} \psi \phi^{2}+C \frac{1}{b^{2} R^{4}}+C \frac{\kappa}{b^{2} R^{2}}
\end{aligned}
$$

and

$$
\begin{aligned}
\left|\psi_{t}\right| \phi & =\psi^{1 / 2} \phi \frac{\left|\psi_{t}\right|}{\psi^{1 / 2}} \\
& \leq \frac{b^{2}}{8} \psi \phi^{2}+\frac{C}{b^{2} T^{2}} .
\end{aligned}
$$

Finally, we have

$$
\kappa \psi \frac{|\nabla u|^{2}}{\left(b^{2}-\rho^{2}\right)^{2}}=\kappa \psi \phi \leq \frac{b^{2}}{8} \psi \phi^{2}+C \frac{\kappa^{2}}{b^{2}} .
$$

From (2.8), (2.9), (2.10), (2.11), (2.12) and (2.13), we obtain that

$$
\psi \phi^{2} \leq \frac{C}{b^{4}}\left(\frac{1}{R^{4}}+\frac{1}{T^{2}}+\kappa^{2}\right) .
$$

For $\psi(x, t)=1$ on $Q_{R / 2, T / 4}$,

$$
\sup _{Q_{R / 2, T / 4}} \frac{|\nabla u|}{b^{2}-\rho^{2}(u)} \leq \frac{C}{b}\left(\frac{1}{R}+\frac{1}{\sqrt{T}}+\kappa\right) .
$$

Proof of Theorem 1.3. Fix $\left(x_{0}, t_{0}\right)$ in space-time and let $f(R)=\sup _{Q_{R, \sqrt{R}}} \rho(u(x, t))$. Applying Theorem 1.2, we deduce that

$$
\frac{\left|\nabla u\left(x_{0}, t_{0}\right)\right|}{4 f^{2}(R)-\rho^{2}\left(u\left(x_{0}, t_{0}\right)\right)} \leq \frac{C}{R f(R)} .
$$

Since $f(R)=o(R)$ by assumption, the result follows after taking $R$ to $\infty$. 


\section{ACKNOWLEDGEMENTS}

This work was carried out at the Beijing International Center for Mathematical Research, China. The author thanks Professor Jiayu Li for suggesting this problem. The author was supported by NSF of China 10701064, 10931001.

\section{REFERENCES}

[1] Cheng, S., Yau, S., Differential equations on Riemannian manifolds and their geometric applications. Comm. Pure Appl. Math. 28.3 (1975), 333-354. MR0385749 (52:6608)

[2] Li, J., Wang, M., Liouville theorems for self-similar solutions of heat flows. J. Eur. Math. Soc. 11.1 (2009), 207-221. MR 2471137 (2009m:58031)

[3] Li, P., Yau, S.-T., On the parabolic kernel of the Schrödinger operator, Acta Math. 156.3-4 (1986), 153-201. MR834612 (87f:58156)

[4] Philippe, S., Zhang, Q., Sharp gradient estimate and Yau's Liouville theorem for the heat equation on noncompact manifolds, Bull. London Math. Soc. 38.6 (2006), 1045-1053. MR2285258 (2008f:35157)

[5] Li, P., Tam, L., The heat equation and harmonic maps of complete manifolds, Invent. Math. 105.1 (1991), 1-46. MR.1109619 (93e:58039)

[6] Hamilton, Richard S., A matrix Harnack estimate for the heat equation. Comm. Anal. Geom. 1.1 (1993), 113-126. MR.1230276 (94g:58215)

Department of Mathematics, Zhejiang University, Hangzhou, 310027, People's RePUBLIC OF CHINA

E-mail address: mathdreamcn@zju.edu.cn 